\title{
Holographic confocal microscope
}

\author{
Irena Antošová, ZdenĚk HaRna
}

Brno University of Technology, Institute of Physical Engineering,

Technická 2, 61669 Brno, Czech Republic, e-mail: antosova@fyzika.fme.vutbr.cz.

\begin{abstract}
We have developed a new laboratory version of a reflected-light holographic confocal microscope (HCM). The confocal imaging capability with the depth discrimination has been verified by measuring the axial intensity response for a perfect plane mirror. The axial resolution is considerably improved without necessity of high-NA objectives using broadband illumination. The operating principle of the mechanisms employed in a laboratory version of the HCM is outlined.
\end{abstract}

Keywords: confocal microscopy, profilometry, 3D imaging, real-time imaging.

\section{Introduction}

Confocal microscopy is a powerful tool that is used in biology, medicine and materials science. Conventional confocal microscopes are based on dual scanning. An image is built up by scanning over the sample by the images of a point source and of a point detector in synchronism. In this way, only the in-focus points of the sample are included in the image. The suppression of the contribution of light from out-of-focus regions of the specimen is the basic feature of the confocal imaging. Only a thin optical section is imaged.

\section{Microscope implementation}

We have developed a new laboratory version of a holographic confocal microscope (HCM) with the depth discrimination based on the broad-source image plane holography [1]. The depth discrimination is achieved owing to the spatially incoherent illumination. Our design arises from a parallel-mode confocal microscope developed by CHMELÍK and HARNA [2].

The significant improvements of the experimental setup proposed below (see Fig. 1) are the following: easy alignment of a microscope, horizontal position of a specimen, the optical system is arranged quasi-symmetrically. 


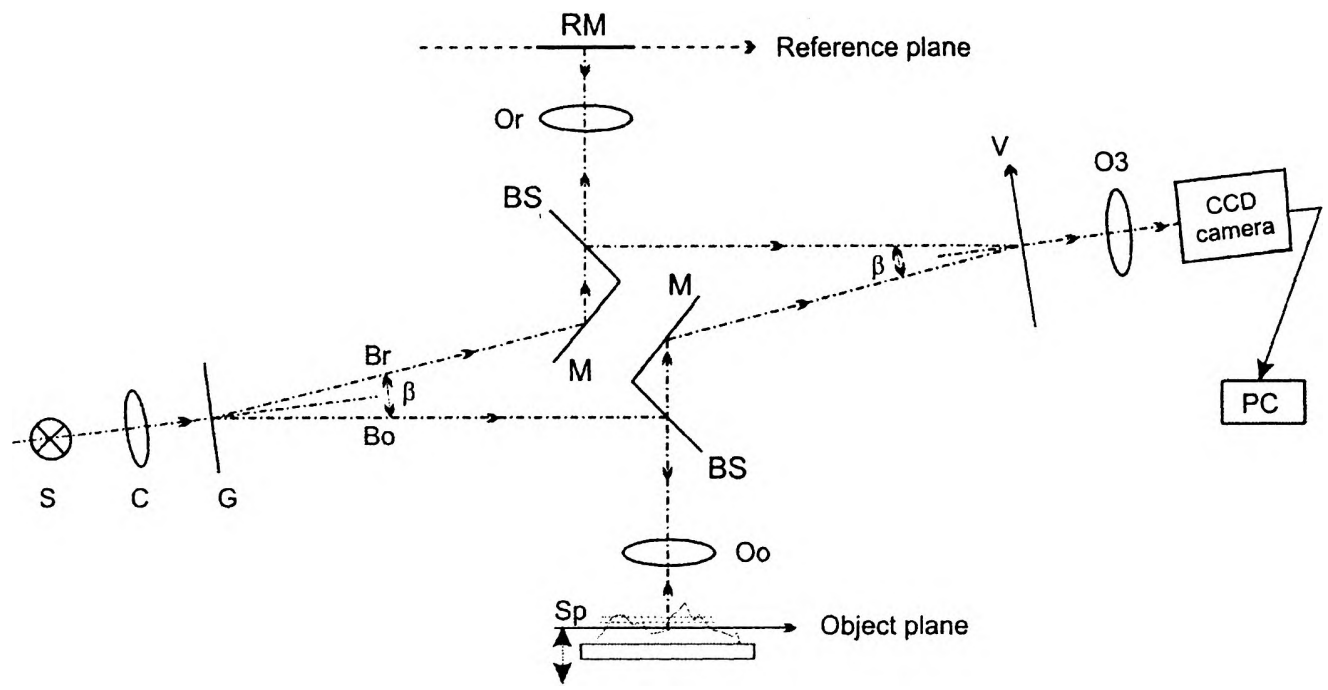

Fig. 1. Experimental set-up of the reflected-light HCM (S - broad source, C - lens, G - diffraction grating, $\mathrm{Bo}$ - object branch, $\mathrm{Br}$ - reference branch, $\mathrm{RM}$ - reference mirror, Oo, Or, O3 - objectives, $\mathrm{BS}$ - beam splitter, $\mathrm{M}$ - mirror, $\mathrm{Sp}$ - specimen, V - output plane).

The experimental setup was designed for wavelength $\lambda=547 \mathrm{~nm}$ and CCD camera with sampling frequency of approximately $110 \mathrm{~mm}^{-1}$, which is sufficient with respect to the maximum frequency observed equal to $40 \mathrm{~mm}^{-1}$ [3].

\subsection{Illumination system}

We use a halogen bulb OSRAM $100 \mathrm{~W}$ providing spatially incoherent light as a polychromatic light source. The broad source (S) is the illuminated spot on a diffuser obtained by the incident light. In conjunction with a narrow-band interference filter we obtain narrow-band illumination at wavelength $\lambda=547 \mathrm{~nm}$ (the full width at half maximum is equal to $30 \mathrm{~nm}$ ). The quasi-monochromatic light source (laser diode FLEXPOINT at $\lambda=655 \mathrm{~nm}, P=20 \mathrm{~mW}$ ) provides spatially coherent light. To achieve confocal imaging, it is necessary to use spatially incoherent light. The most effective way of converting spatially coherent light into spatially incoherent one is to introduce a two diffuser system, a rotating diffuser associated with a motionless diffuser, inserted to the laser beam [4]. The light spot on the rotating diffuser surface created by the light passing through is the broad source of illumination.

\subsection{Experimental setup}

The fundamental part of the microscope consists of a diffraction grating $(\mathrm{G})$, two identical beam splitters (BS), two mirrors (M) and two identical achromatic microscope objectives (Oo and Or 10/0.25), which simultaneously illuminate and 
image the specimen $(\mathrm{Sp})$ and the reference mirror $(\mathrm{RM})$. The primary beam emitted by the broad source $(S)$ is split by the linear phase diffraction grating $(\mathrm{G})$ into two branches of the interferometer, into the object branch (Bo) and reference branch $(\mathrm{Br})$, using the \pm 1 diffraction orders. Thus two identical optical branches are introduced. The broad source is imaged to the image focal planes of the objectives Oo and $\mathrm{Or}$ by the doublet $\mathrm{C}$. In this way the Köhler illumination (i.e., uniform illumination) of the specimen and of the reference mirror in the object planes of Oo and $\mathrm{Or}$ is ensured.

\subsection{Wave propagation in the optical system}

Let us make the following simplifying assumptions: the influence of the diffraction is neglected and a point is approximately imaged into a point. The primary images of the broad source are formed in the image focal planes of the objectives (Oo and Or). We suppose that any two points of the source image are mutually spatially incoherent and simultaneously each point of the broad source is imaged into two points, which are mutually coherent. Divergent spherical waves, which spread out from all points of each source image, are transformed by the objective into plane waves. In this way, the Köhler illumination (i.e., uniform illumination) is ensured. The plane waves are reflected by the reference mirror and by the sample and then focused back to the focal planes. In this way, the secondary images of the source are formed. The reference wave, which spreads out from the focal plane of the image space, is a divergent spherical wave of a known form. We can find the form of this object wave because it characterizes the sample being observed. The reference wave and the object wave, which both spread out from one point of the broad source, interfere with each other and form the image-plane holographic pattern in the output plane (V).

The beam splitters and mirrors change the direction of beam propagation so that the angular distance of the secondary images with respect to the output plane equals the angular distance of the primary images with respect to the diffraction grating for each wavelength of illumination.

The holographic pattern with the spatial carrier frequency, which equals double the grating frequency, is formed in the output plane for each wavelength of illumination. Thus the interferometer is achromatized and confocal imaging is achieved with broadband illumination. The interference condition is satisfied only for the light, which is reflected by the in-focus section of the specimen. The holographic pattern is imaged by the objective (O3) onto the $\mathrm{CCD}$ chip and the image amplitude and phase are reconstructed using fast Fourier transform algorithm.

\section{Experiment}

The experiments were performed with a laboratory version of the HCM, which was constructed in accordance with the previously proposed experimental setup. An 
interference pattern was scanned by a CCD camera WAT $902 \mathrm{H}$ with a $2 \mathrm{D}$ array of $811 \times 508$ pixels. The image processing was performed by the Matlab procedure.

\subsection{Alignment}

The purpose of this part is to outline the operating principle of the mechanisms employed in a laboratory version of the HCM. The aim of the project is to develop a microscope that is easy to align and consequently capable of producing sufficiently fine reproducible movements.

The mirror and the beam splitter in the object branch are fixed. The sample is mounted in the linear micropositioner designed by ANTOš [5], which allows shifting the sample against the objective Oo in three independent axes of an orthogonal coordinate system. The $x-y-z$ translation mechanism is created by the parallelogram with flexure pivots. The micropositioner is fixed on a piezo-electric transducer. The displacement of the piezoelectric stack is limited to within $20 \mu \mathrm{m}$.

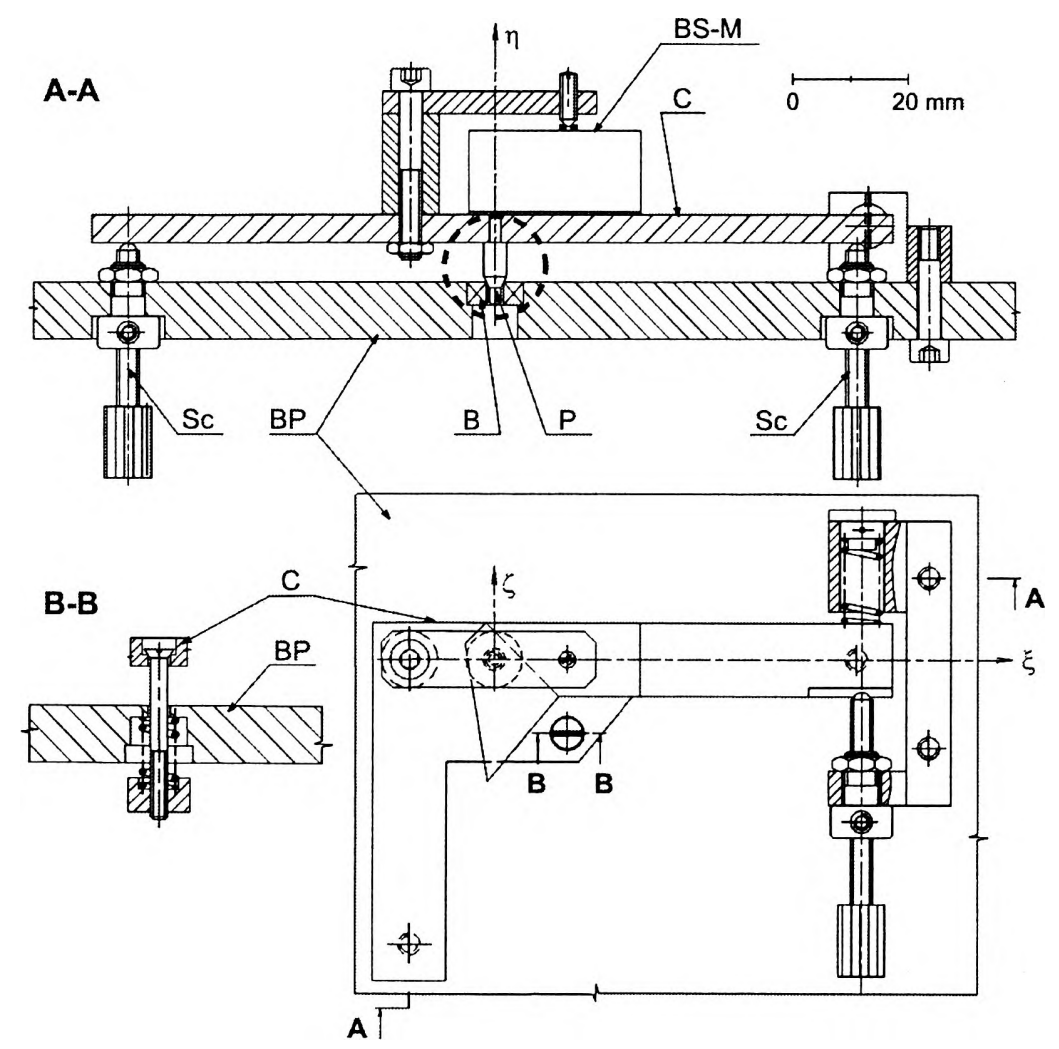

Fig. 2. Scheme of the micropositioner, which carries the mirror (M) and the beam splitter (BS) in the reference branch (BP - base plate, $\mathrm{C}$ - carrier, $\mathrm{B}$ - ball bearing, $\mathrm{P}$ - pivot, $\mathrm{Sc}$ - fine-pitch screw). 


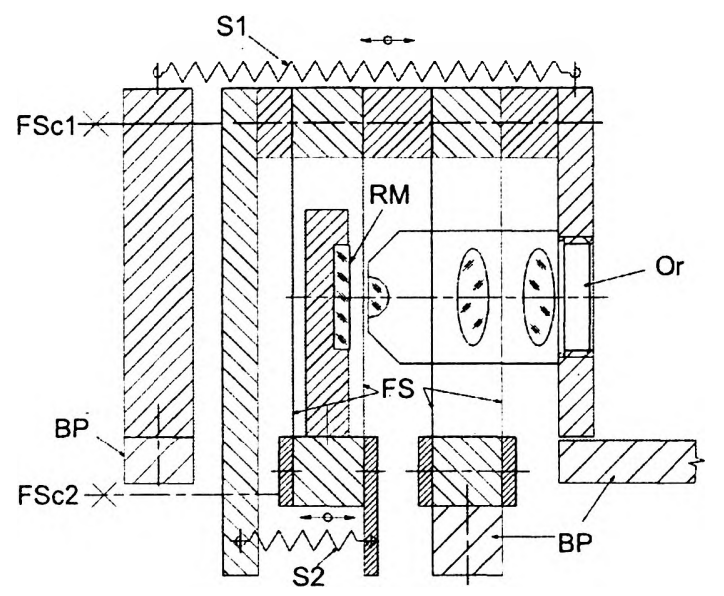

Fig. 3. Arrangement of the mechanical system for positioning the reference mirror (RM) and the objective (Or); (FS - flat springs, BP - base plate, S1, S2 - extension cylindrical springs, FSc1, FSc2 - fine-pitch screws).

The mirror and the beam splitter in the reference branch are mounted in the micropositioner (see Fig. 2), which allows rotation in three independent axes $\xi, \zeta, \eta$ of an orthogonal coordinate system so that both images appear in the output plane in an identical position.

The reference mirror (RM) and the objective Or are carried by a flexure-pivoted mechanical system (see Fig. 3) that allows a shift of the mirror against the objective. In this way the image is focused to the output plane. Shifting simultaneously the objective and the mirror we set the same optical path in both the reference and the object branch. When the microscope is aligned, high contrast interference fringes appear in the output plane.

\subsection{Axial intensity response of the microscope}

The axial intensity response is the rate at which the detected intensity falls off with defocusing. When a point source of illumination, a point detector and aberation-free optics are assumed, the axial intensity response of a conventional confocal system is given by the expression [6]

$$
I_{r}=\left\{\frac{\sin [2 \pi k z(1-\cos \alpha)]}{2 \pi k z(1-\cos \alpha)}\right\}^{2}
$$

where $\alpha$ denotes the angular aperture of the objectives, $k=1 / \lambda$ is the wave number ( $\lambda$ is the wavelength of the illumination), and $z$ is the defocus of the reflector. 


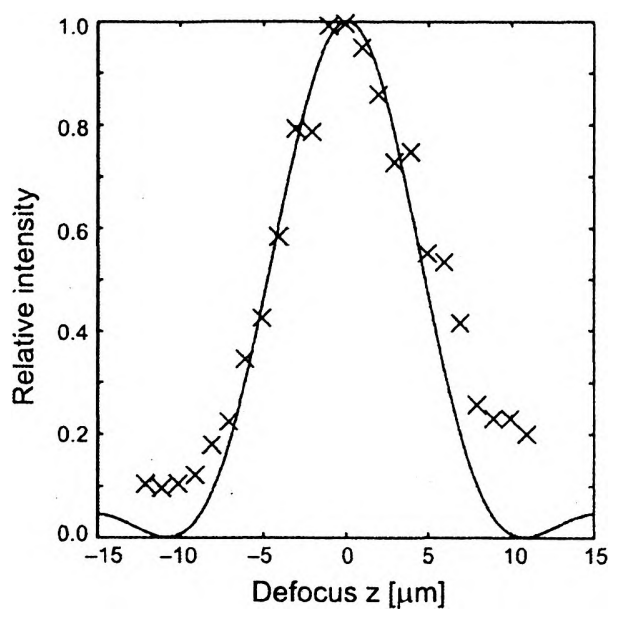

Fig. 4. Axial intensity response for a plane mirror; $10 / 0.25$ achromatic objectives, quasi-monochromatic illumination $(\lambda=655 \mathrm{~nm})$. In practice, we scanned a perfect plane reflector axially through focus with the step $1 \mu \mathrm{m}$ and registered the form of the response. The theoretical axial intensity response (solid curve) was evaluated according to Eq. (1), the experimental axial intensity response of HCM is marked by crosses.
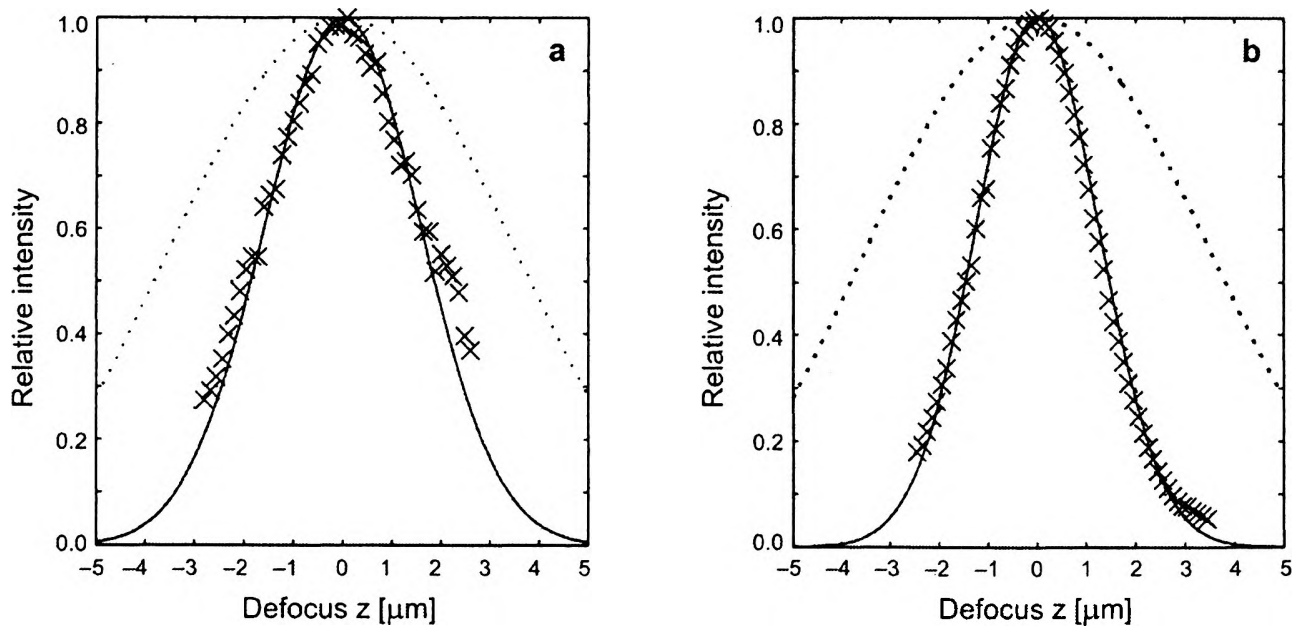

Fig. 5. Axial intensity response for two differrent spectral densities of illumination; $10 / 0.25$ achromatic objectives, $\mathbf{a}$ - narrow-band illumination $\lambda=547 \mathrm{~nm}$, and spectral half width $\Delta \lambda=30 \mathrm{~nm}$, $\mathbf{b}$ - white-light illumination. In practice, we scanned a perfect plane reflector axially through focus with the step $130 \mathrm{~nm}$ and registered the form of the response. The theoretical axial intensity response is plotted as a solid curve, the theoretical axial intensity response for monochromatic illumination $(\lambda=547 \mathrm{~nm})$ is plotted as a dashed line and the experimental axial intensity response of HCM is marked by crosses. 
The theoretical axial intensity response is plotted as a solid curve in Fig. 4. The full width at half maximum is described by the expression [6]

$$
\mathrm{FWHM}=2 z_{1 / 2} \approx \frac{0.443 \lambda}{1-\cos \alpha} .
$$

The pinhole size in a real confocal microscope is of the order of $10^{0}-10^{1} \mu \mathrm{m}$. Therefore, the FWHM has a higher value. In accordance with Eq. (2), the axial resolution can be improved using high-NA objectives. The axial intensity response of conventional light microscope stays $I_{r}=1$.

We have verified the confocal imaging capability with the depth discrimination by measuring the axial intensity response for a plane mirror (see Figs. 4, 5). The imaging parameters of $\mathrm{HCM}$ for monochromatic illumination correspond to the imaging by the conventional confocal microscope. The axial resolution can be considerably improved without the necessity of high-NA objectives using broadband illumination [7]. The improvement of axial resolution due to spectral broadening has also been verified. The theoretically predicted axial responses are in a good agreement with the experimental data (see Fig. 5).

\section{Conclusions}

We developed a new laboratory version of the reflected-light holographic confocal microscope. We have verified the confocal imaging capability with the depth discrimination by measuring the axial intensity response for a plane mirror. The postulated optical system of the holographic confocal microscope has no scanning system. All object points of the whole viewed field are imaged simultaneously. Hence, the real-time image acquisition is achieved. The imaging speed is limited only by the image detection and recording process. Both the image amplitude and the image phase are reconstructed from the holographic signal in the output plane. Thus, the entire confocal image information is acquired. The possibility of reconstructing the image phase provides an enhancement of the depth resolution. The image phase varies according to the equation $\varphi=2 \pi k z(1+\cos \alpha)$ [8], the depth resolution is within the range of a few nanometers.

The system is not able to produce fluorescence imaging due to the loss of coherence in the fluorescence process. Objects in dispersive media have to be imaged using laser illumination for the same reason. The field of view is relatively small compared to conventional microscopes.

A suitable application arises especially in materials science for noncontact and nondestructive surface profiling of reflective materials. The nanometer-scale measurement of surface profiles is possible due to the image-phase processing. The HCM is suitable for imaging the living tissues owing to nondestructive optical 
sectioning capability, for observing: rapidly changing phenomena, surfaces covered by transparent layers, wear, fracture surfaces, samples made by lithography, integrated circuits, and so on.

\section{References}

[1] Sun P.C., Leith E.N., Appl. Opt. 33 (1994) 597.

[2] Chmelik R., Harna Z., Opt. Eng. 38 (1999), 1635.

[3] Webs R.H., Dorey C.K., The Pixilated Image, Handbook of Biological Confocal Microscopy, 2nd edition, [Ed.] J.B. Pawley, Plenum, New York 1995, pp. 55-67.

[4] Lowenthal S., Joyeux D., J. Opt. Soc. Am. 61 (1971), 847.

[5] Antoš M., Harna Z., Jemná mechanika a optika, 44 (1999), 352.

[6] Jordan H.J., Wegner M., Tiziani H., Meas. Sci. Technol. 9 (1998), 1142.

[7] Chmelik R., Harna Z., Jemná mechanika a optika, 46 (2001), 127.

[8] Chmelik R., Harna Z., Opt. Eng. 41 (2002), 744. 